\title{
Let Me Show You Your New Home: Studying the Effect of Proxemic-awareness of Robots on Users' First Impressions
}

\author{
Björn Petrak*, Katharina Weitz*, Ilhan Aslan*, and Elisabeth André*
}

\begin{abstract}
First impressions play an important part in social interactions, establishing the foundation of a person's opinion about their counterparts. Since interpersonal communication is essentially multimodal, people are judged during first encounters by both their verbal utterances and nonverbal behavior, such as how they utilize eye contact, body distance, and body orientation. In this paper, we argue that robots would provide better user experiences, including being perceived as more likable if they were able to make a good first impression when introduced to a new home. Moreover, we wanted to test if robots can improve their perceived impression by behaving in a proxemic-aware manner; i.e., by following established social norms, which prescribe, for example how far people should position themselves around other objects to improve the facilitation of social interactions. In order to test this hypothesis, we conducted a user study with 16 participants in a virtual reality setting, comparing the impression of two agents being introduced to their new homes by users. We found that the proxemic-aware agent was indeed perceived as significantly better considering multiple constructs, including perceived anthropomorphism and trustworthiness.
\end{abstract}

\section{INTRODUCTION}

The field of personal service robotics in particular is seen as having growth potential for the future [1]. Here, robots can enrich people's lives by providing physical support (e.g., as support for housework) or address psychological aspects (attention \& caring, support for social behavior, coaching) [1]. These tasks can be covered in varying levels of complexity by a robot. The spectrum ranges from very mechanical and seemingly not very lively machines that perform a specific task (e.g., vacuum cleaner robots) to robots that are perceived as living entities. With these living entities, the scope of action covers very simple, non-verbal and intuitive ways of acting, as one would ascribe them to pets. But also very complex robots, which express themselves through language and appear at least human-like, can be in focus (e.g., Sophia ${ }^{1}$ ). In order to be able to use social robots for tasks in the private environment, technically challenging requirements such as cognition (planning \& decision making), perception (navigation \& environment sensing), and action (e.g., mobility \& manipulation) [2] have to be solved. In addition to these technical challenges, the aspect of human trust in the robot must also be included in the design of these systems. The first contact between human being and robot is a special situation in which technical aspects have to prove themselves. This first contact is also an important moment in the trust-based relationship between robot and human

\footnotetext{
*Human-Centered Multimedia Lab, Augsburg University, Germany lastname@hcm-lab.de

${ }^{1}$ https://www.hansonrobotics.com/sophia/
}

being. After purchasing a new social robot and unpacking it at your home, the robot is in an unknown location in an unknown environment. One of the robot's first tasks will therefore be to discover its environment and create a map of it so that the robot can navigate autonomously around your home [3]. This paper is about the consideration of this first contact. The focus is on the technical implementation of the perception aspect, especially the aspect of navigation. In the following study, we compared participants' evaluation of two different behavior of robots in virtual reality (VR) as a prototyping method, while exploring an unknown room. One robot's behavior is proxemic-aware (i.e., looking after the user during the exploration) and the other robot is exploring the room on his own as a baseline condition. The two robots are deliberately kept simple and pet-like in their behaviour and communication skills.

We argue that the proxemic-aware behavior of the robot results in people perceiving the robot to be more anthropomorphic (H1a) as it copies the natural spatial behavior of humans and also leads to higher ratings in animacy (H1b). In addition, we expect that the behavior of the robot increases likability (H1c) and that the robot is perceived as more intelligent (H1d). Furthermore, addressed the aspect of human-robot trust in this situation. We expect participants to have more trust in a robot (H2a) when it explores the room together with the user and users will be more interested in interacting again $(\mathrm{H} 2 \mathrm{~b})$ with the robot in the future.

Before we describe the VR setting, the user study, and present all results in detail, we provide a brief background of proxemic interactions and trust in a human-robot relationship.

\section{BACKGROUND AND RELATED WORK}

\section{A. Proxemics}

Proxemics is a term defined by Edward Hall [4] to describe the spatial behavior of individuals. He describes proxemics as a non-verbal and implicit form of communication, i.e. how people use the space around them and how it influences interaction and communication with other people in the nearby space. Research in human-robot interaction is often inspired by Hall's theory. This results in a lot research on how robots and humans approach each other and finding the right distance (e.g., [5], [6], [7], [8]), or how sociallyaware navigation can be implemented while taking proxemic conventions into account [9].

Marquardt and Greenberg [10] propose the Proxemic Interaction Framework for the realization of proxemic behavior in human-computer interaction. For the framework they 
identified the dimensions distance, orientation, movement and motion, identity, and position as relevant for the spacial interactions.

Researchers have adopted a reactive and proxemic interaction design approach in diverse fields and explored for example proximity-sensitive actuated and shape-changing mobile phones (e.g., [11], [12]) and proxemic touch screen targets (e.g., [13],[14],[15]). Common to the aforementioned examples is that the research didn't focus specifically on anthropomorphism, but still report that proxemic behavior resulted in users referring to interfaces and interface elements as if they were living organism and animals with agency and a consequent increase in perceived hedonic qualities (e.g., fun). While stationary computer systems e.g. in smart homes only are able to react to proxemic behavior of users (e.g., [16]), virtual agents can at least use their body orientation and gaze behavior (e.g., [17]) to show proxemic behavior. Bee et al. [18] used only the orientation dimension utilizing gaze in a "first impression scenario" to get into interaction with a human. But mobile robots are enabled to not only use this one dimension, but also to move in a room by themselves. They can therefore not only react to proxemic behavior of humans, but they can also show active spatial behavior in multiple dimensions and thus enable a wider range of interaction possibilities. In a recent work $\mathrm{Li}$ et al. [19] have taken inspiration from improv theater studied how a non-anthropomorphic robot can use locomotion only for social expression (i.e., dominance). In sum, related work strongly indicates that a mobile companion robot, which acts in a proxemic-aware manner will result in users perceiving them as more alive, experience more hedonic qualities, and ultimately the robot will leave a better first impression than a version which is mobile and autonomous but not proxemic.

Hall's notion of proxemics does not fully describe the spatial behavior between different co-located persons. One description of spatial arrangement in small groups is the theory of f-formations [20]. Typically, communication in groups of 2-5 people takes place in a circular cluster. The area in the middle of the circular arrangement of persons is called o-space and is reserved for the main activity of the group. An object within the o-space becomes part of the group conversation. The ring around the o-space, in which the persons are located is called p-space. The region outside these zones is called r-space. Depending on the body orientation of the participants, a distinction is made between different types of f-formations. For example, there are faceto-face, side-by-side or corner-to-corner (i.e., the persons are at right angles to each other) f-formations.

\section{B. Social Interaction \& Trust}

Trust is a fundamental basis for the development of socially interactive robots that will be used in the private environment (e.g., [21], [22]). When talking about (social-) interaction in robotics, it has to be differentiate between two kinds of interactions: robots as collective interactors [23], [24] or as individual interactors [25]. Collective interactors describe societies which are characterized by being anony- mous, homogeneous groups [2]. Dautenhahn and Billard [25] defined social robots as individual interactors, who are part of a heterogeneous group and are perceived as individuals. To model and develop social robots, social learning and imitation, gesture and natural language communication, emotion, and recognition of the interacting partners have to be considered [2]. Coeckelbergh [26] points out that the appearance of the robot has an influence on trust of people. It has been shown that people tend to interpret active body movements as a sign of sympathy for others [27]. Therefore, the perception of the robot as trustworthy is influenced by its movement behaviour [28]. For example, the investigation of the proxemic behaviour of robots in the household environment in the work of Oviatt et al. [29] showed that proxemics can have an influence on the user's trust. Likewise, the amount of trust people place in the robot has an impact on how they evaluate the robot's proxemic behavior [30]. The perception of the movement behaviour of the robot is part of an affective component which is involved in the evaluation of the robot as trusting. This corresponds to the definition of Lewis and Weigert [31] which divide trust into cognitive and affective aspects. In the context of humanrobot trust, cognitive trust can be seen as mental attributes, reasons and arguments of a person towards an agent, whereas affective trust describes the feeling of a person towards an agent [32].

\section{VR PROTOTYPE/ENVIRONMENT}

In order to test our hypotheses, we created a virtual setting in which we conduct a user study. In the following we describe the environment (i.e, the room) in which the user is placed and the robots the user interacts with.

\section{A. Room}

When users put on the VR headset, they are in a room that looks like a living room. The room measures approx. $2.5 \mathrm{~m} * 5.5 \mathrm{~m}$ and users can move around freely, as the dimensions correspond to the room in which the user physically is. The virtual room is filled with some furniture (e.g. tables, shelves) and several objects (e.g. TV, flowers) as shown in Figure 1. So that both, robot and user, can move easily in the room, we have positioned the interior only at the sides of the room.

\section{B. Robots}

Together with the user there is one of two robots in the room, which have the same design, but differ in their behavior. The robots have a simple structure and consist of a torso, a head with eyes and nose, and arms. The main purpose of the arms is to help the user see in which direction the robot is oriented. The robots are about $1 \mathrm{~m}$ tall and "glide" over the floor as if they were moving on wheels, similar to the robot Pepper $^{2}$. We decided to keep the robots simple so that the users always know where the robot is looking and in which direction its body is oriented, and we gave him a smaller height to give him a pet/companion look.

${ }^{2}$ https://www.softbankrobotics.com/emea/en/pepper 


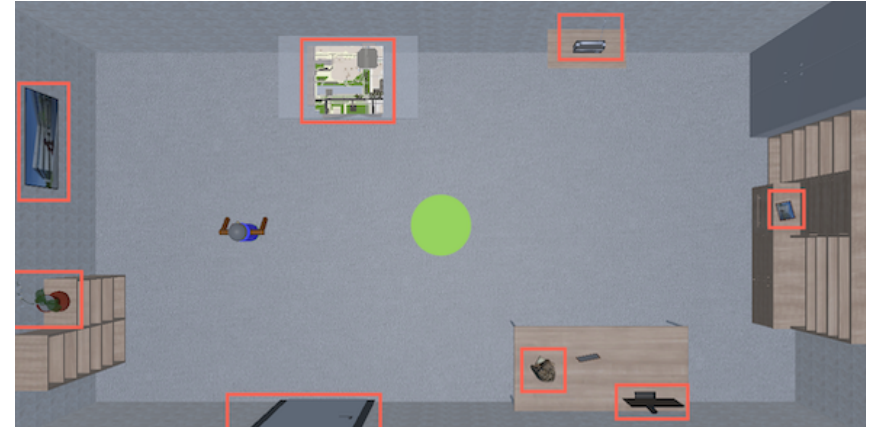

Fig. 1. Top view of the room in which the interaction with the robot takes place. The green dot shows the user's entry point. Red outline highlights intractable objects, clockwise from bottom left: flower, TV, map, radio, picture, monitor, statue, door.

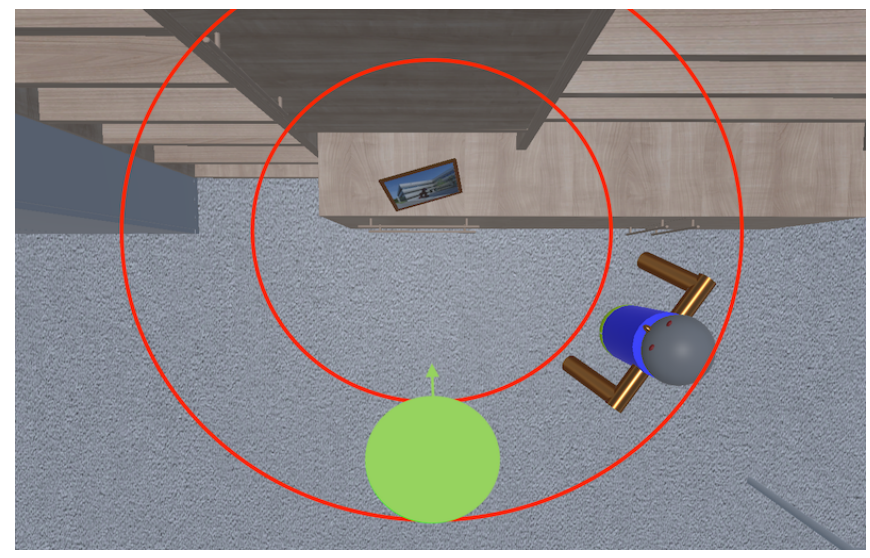

Fig. 2. The positioning of the robot in the f-formation while the user is looking at an object in the room. The object (i.e., the picture) is in the o-space (inner red circle), while the person (green circle) and robot are in the p-space (between the two red circles).

The behavior of the two robots is guided by the goal of exploring the objects in the room. The proxemic-considering robot tries to explore the objects together with the user, while the other robot explores the room autonomously. The behavior has been defined using the Proxemic Interaction Framework [10]. We manipulate the spatial behavior of the robots in the proxemic dimensions location (i.e., where in the room the robot is positioned) and orientation (i.e., body and head orientation).

When the user approaches and orientates towards an object, the robot positions itself in an "corner-to-corner" fformation together with the user in the p-space, while the object is in the o-space so that they look at the object together (see Figure 2). Depending on where there is more space, the robot can position itself to the right or left of the user. When the user faces the robot, the robot faces the user and looks at him. The robot adjusts its body orientation as well as the head direction in such a way that it looks at the object or at the user's eyes (i.e., in the direction of the camera). Figure 3 shows the robot's orientation behavior from the user's point of view when looking at an object or at the robot. As soon as the user moves on to the next object, the robot follows and repositions itself according to the same rule for the new object.

The behavior of the autonomous robot is defined in that it randomly selects an object in the room, approaches it and orientates itself towards the object. The robot then looks at the object for a few seconds before randomly selecting a new one. The robot "ignores" the user completely.

\section{STUDY}

\section{A. Design and Participants}

A total of 16 participants took part in the within-subjects study, most of them without any experience in interacting with robots. Half of the participants were female and the other half male and a majority were between 20 and 30 years old.

\section{B. Procedure}

Participants were mainly German students recruited at a university. We told the participants that the study is about interaction with two robots that have different ways of interaction. They were given the task to look around the room and search for objects (e.g., picture, statue), so that the robot learns where the objects are.

At the beginning, the participants read the instructions and then explored the room with both robots in a counterbalanced order in the virtual environment. We helped the participants to put the VR headset (HTC Vive Pro ${ }^{3}$ ) on correctly, so that the image was clearly visible and the headset fitted comfortably. When the participants put the headset on correctly, they explored the room, with each run taking about four to six minutes. We let the participants interact with the robot as long as they wanted. After taking the headset off again, they rated the robot (see subsection IV-C. Dependent Measures) and then repeated the procedure with the other robot. Once they interacted with both robots and finished the rating, they selected which robot they preferred to interact with and gave reasons for their choice.

\section{Dependent Measures}

The German version of the Godspeed questionnaire series [33] was used to evaluate the impact of the two robots on the user. This questionnaire is a validated tool for measuring human-robot interaction (HRI). It comprises five core concepts of HRI: anthropomorphism, animacy, likeability, perceived intelligence, and perceived safety that are queried using different semantic differentials. Participants must evaluate each semantic differential on a scale from 1 to 5 (e.g., for the concept of animacy: dead vs. alive). Due to the research questions described in I, only four of the five concepts queried in the Godspeed questionnaire series were used for the evaluation. The evaluation of the concept perceived safety was not part of the research questions and therefore also not part of the evaluation.

In order to get an impression of the effect of the two robots, the participants were also asked how trustworthy they considered the respective robot to be and whether they would

${ }^{3}$ https://www.vive.com/us/product/vive-pro/ 

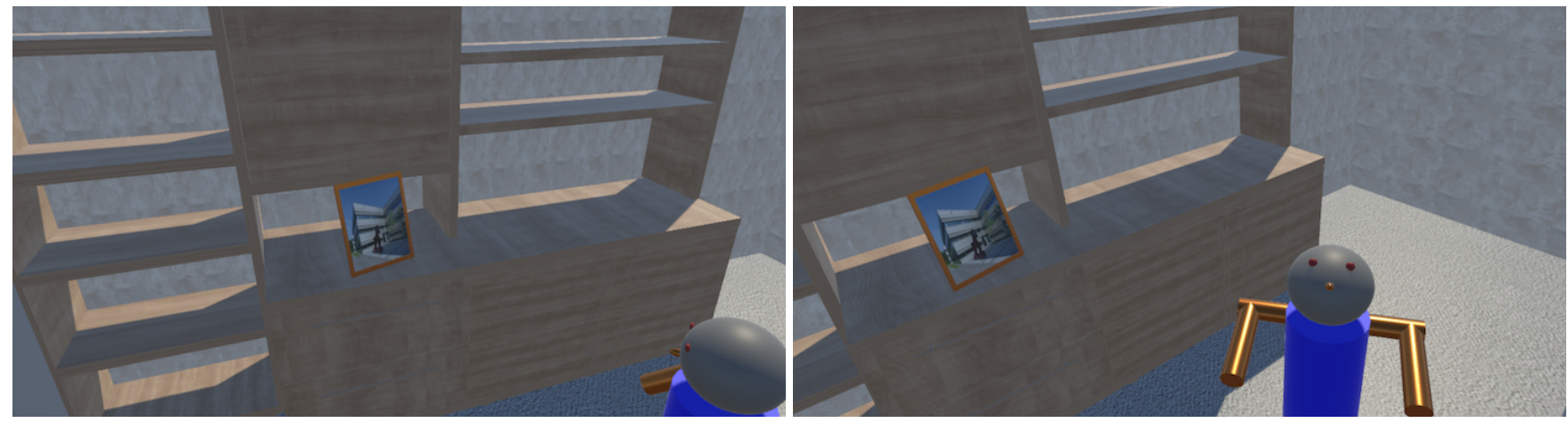

Fig. 3. Robot orientation behavior. Left: When the user looks at an object. Right: When the user looks at the robot.

interact with the robot again. These two questions were asked using a 5-point Likert scale (-2=disagree, $2=$ fully agree).

In order to gain even more information about what played a role in the participants' evaluation of the robot, open questions were also asked about what the participants found to be particularly good and particularly bad about the respective robot. At the end of the study, the participants also had to reevaluate which robot they preferred and justify their answer.

\section{Results}

First, the general trends in the quantitative data, collected with the Godspeed questionnaire series and the two items evaluating the trustworthiness in the robot and desire for re-interaction with the robot were analyzed. Afterwards, we describe the results of the qualitative data (open questions) analysis aiming to provide reasons for specific results and observations.

\section{A. General Trends}

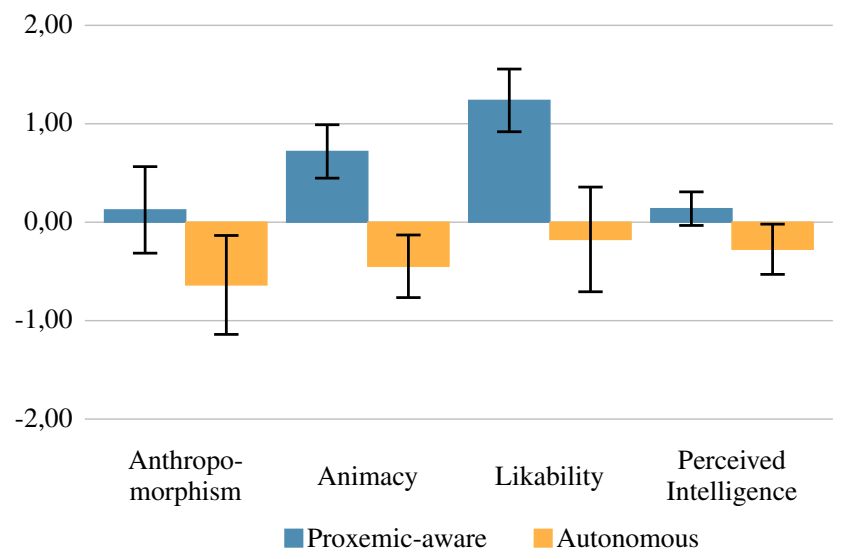

Fig. 4. Overview of mean values for each condition and dependent variable measured by the Godspeed questionnaire series. Error bars denote 95\% confidence intervals. Scale ranges from -2 to 2 .

Figure 4 and figure 5 graphically present the mean values of the dependent variables measured. When looking at the plots it is noticeable that in all areas the proxemic-aware robot is rated higher than the autonomous robot. Furthermore, all ratings of the proxemic-aware robot are in the positive range of the scale $(0-2)$, while all ratings of

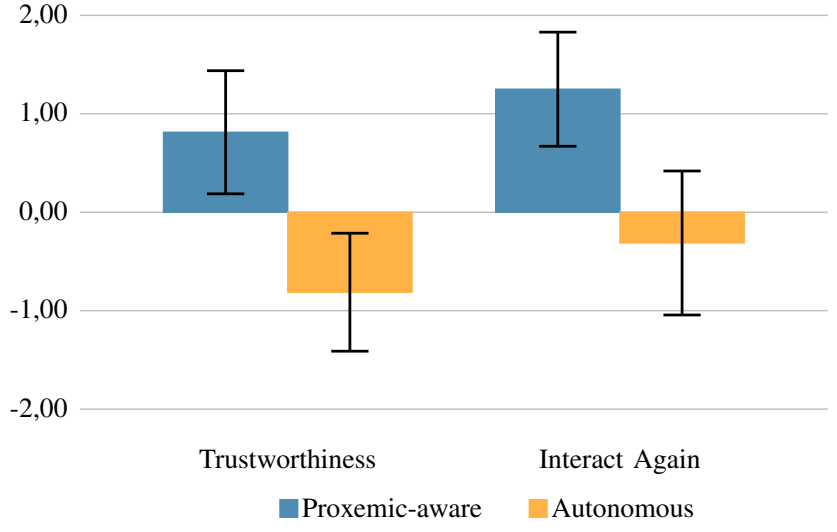

Fig. 5. Overview of mean values for each condition and dependent variable measured by the questions on . Error bars denote $95 \%$ confidence intervals. Scale ranges from -2 to 2 .

the other robot are in the negative range $(-2-0)$. The biggest differences in the rating are on the scales likeability, trustworthiness, and whether users would interact with the robot again.

\section{B. Statistical Analysis}

A paired-samples t-test was conducted to test the differences between the two robots (i.e., proxemic-aware, autonomous) across the dependent variables measured by the Godspeed questionnaire series (i.e., anthropomorphism, animacy, likability, perceived intelligence) and the two additional questions regarding trust and if the people would interact again with the robot. Table I shows the mean values and the standard deviation of the dependent variables and the results of the t-tests with corrected p-values for multiple testing using the Holm correction [34]. The conducted t-tests showed significant effects for the four relevant concepts of the Godspeed questionnaire series. In order to estimate the size of the effect, hedges $g^{*}$ were calculated in which the variances are not only pooled but also corrected with the Bessel correction, thus reducing the error of estimating the effect strength, especially in small sample [35]. In addition, the use of $g^{*}$ corrects the positive approximation error that occurs when calculating $g$ [36]. According to Cohen's [37] recommendation, the effect strengths can be interpreted as 
follows: Values above 0.2 speak for a small effect, values above 0.5 for a medium effect and values above 0.8 for a large effect.

It was found that the proxemic-aware robot was perceived as more anthropomorphic ( $g=0.77$ - medium effect), more animalistic ( $g=1.89$ - large effect), more likeable ( $g=1.54-$ large effect), and more intelligent $(g=0.87-$ large effect) than the autonomous robot. Additionally, the participants rated the proxemic-aware robot as significant more trustworthiness than the autonomous robot $(g=1.26-$ large effect). There was also a significant difference in the question whether the participants would interact again with the robot, where the participants would rather interact again with the proxemic-aware robot than with the autonomous robot $(g=0.90-$ large effect).

TABLE I

COMPARISON OF PARTICIPANTS' RATINGS OF THE ROBOTS.

\begin{tabular}{lcccccc}
\hline Item & $M_{P}$ & $S D_{P}$ & $M_{A}$ & $S D_{A}$ & $t(15)$ & $p$ \\
\hline Anthropomorphism & 0.13 & 0.90 & -0.64 & 1.03 & 3.09 & $.007^{*}$ \\
Animacy & 0.72 & 0.55 & -0.45 & 0.65 & 7.56 & $<.001^{*}$ \\
Likeability & 1.24 & 0.65 & -0.18 & 1.09 & 5.14 & $<.001^{*}$ \\
Perceived Intelligence & 0.14 & 0.35 & -0.26 & 0.52 & 2.55 & $.011^{*}$ \\
Trustworthiness & 0.81 & 1.28 & -0.81 & 1.22 & 4.61 & $<.001^{*}$ \\
Interact Again & 1.25 & 1.18 & -0.31 & 1.49 & 4.75 & $<.001^{*}$ \\
\hline${ }^{*} p<0.05$, one-sided & $M_{P}=$ proxemic-aware robot, $M_{A}=$ autonomous robot
\end{tabular}

\section{Analysis of qualitative data}

13 of the 16 participants $(81 \%)$ said they preferred the proxemic-aware robot. Reasons why they preferred this robot were that it was "polite", "respectful", "friendly", "courteous", "interested", and that it showed "intuitive behavior". Many participants highlighted in particular that the robot looked back when they looked at the robot and looked at the objects that the user found interesting. In addition, the experience of exploring "together" with the robot was perceived as positive. Also, the behavior of the proxemicaware robot was perceived by participants similar to that of a pet and two of them said the robot was "like a faithful dog". In contrast, the behavior of the autonomous robot was described as "cat-like" in a negative way, since the robot acts independently and does not pay attention to the user. The fact that the robot does not involve the user in the exploration was perceived by the participants as a very negative experience. Participants who preferred the autonomous robot argued that the proxemic-aware robot, although they liked its behavior sometimes, was too intrusive in the long-term and one participant even found the behavior too intrusive right from the beginning.

\section{DISCUSSION}

Overall, the motivation of improving social robots' interaction qualities is that users would benefit greatly if their robots were able to demonstrate social and affective skills, even if skill levels were at the level of a pet [38]. But in fact, many pets such as dogs or cats make use of both appropriate explicit and implicit interaction capabilities and indeed can be socially very smart and foster healthy interactions and relationships with their owners. However, owning or caring for a pet is not an option for many people (e.g., due to allergies and job conditions). In contrast to pets, social robots have a great potential to address the needs of divers users and potentially less ethical consideration need to be addressed (e.g., screaming to a robot may be ok).

We have motivated our research by emphasizing that for social robots and especially companion robots in homes it is important to follow established social norms in interpersonal interaction [39]. These are important to generate positive effects in the field of affective trust and thus to establish and maintain the acceptance of social robots in the private environment. The ongoing proliferation in the field of robotics inspires to explore interaction scenarios, which convey to social norms in an increasingly multimodal and comprehensive manner.

Thus, when social companion robots turn mobile, they should follow the social conventions identified by proxemics, which describes a set of interrelated communication modalities in interpersonal interaction often utilized for implicit spatial interactions, including gaze, body orientation, and spatial positioning.

While it seems reasonable for a social mobile robot to obey the rules of proxemics, it is not necessarily clear how users would perceive such a non-human mobile agent with its potentially peculiar body features. But, because many humans have functioning social relationships with their pets and social robots for homes are often imagined to fill in a similar role and even look and feel similar to a pet, we were optimistic to observe mainly positive effects. In retrospective a socially intelligent and anthropomorphic moving robot could have triggered an uncanny valley effect.

In our study, we focused on an introduction and house tour scenario as a viable future situation in which a mobile companion robot has the opportunity to leave a positive first impression on their future human companions by utilizing and demonstrating proxemic-awareness. We believe that the scenario was well suited to explore the effect of proxemics.

Results of the self-reported data support all our hypotheses (H1a-d, and H2a-b) that a proxemic-aware robot is perceived superior, considering user experience factors, such as likability (see Table I). Participants' reports also show that the they found the proxemic-aware robot as significantly more trustworthy and reported a significantly higher willingness to interact with the proxemic-aware robot. The effects of proxemic-aware behavior on perceived anthropomorphism and animacy confirm our assumption that participants would perceive the proxemic-aware robot due to its behavior as more human-like, animal-like, and thus, as more "alive".

Although the results seem to demonstrate a clear preference (i.e., 13 of 16 participants) towards the proxemic-aware robot, the comments of some participants (especially those who did not prefer the proxemic-aware robot) suggest that there is still space for improvements, which we believe is ultimately due to individual differences in how people perceive proxemic behavior. As aforementioned in the analysis of the interview data, some participants stated that they could 
imagine that the proxemic robot's behavior may become too intrusive or annoying over time. We believe that such individual differences and an adaptation over time can only be addressed by advanced programming approaches, such as reinforcement learning approaches (e.g., [40], [41], [42]).

Overall, the main effect of proxemic-aware behavior on all relevant items of the Godspeed questionnaire strongly implies that robot creators should implement proxemic awareness in their future mobile home companion robots and that by doing so they will create more trustworthy robots with which inhabitants are willing to interact significantly more often.

\section{LIMITATIONS AND FUTURE WORK}

Our research faces some limitation, which fellow researchers should consider when reusing them or applying them to other setups. First, we utilized virtual reality and applied an experience prototyping approach, since it would have been difficult to build the required behavior recognition and reactivity with a physical robot. Participants may perceive both robot behaviors, which we analyzed in the virtual reality setting in reality stronger to the materiality of physical robots and safety concerns that exist in reality but not apply to virtual reality. In our future work, we intent to replicate some of the interaction aspects which we studied solely in virtual reality with the physical Pepper robot. Furthermore, motivated by the results of the study at hand an important focus of our future work will be the research of how proxemic-aware behavior can be taught to mobile companion robots using interactive machine learning and especially reinforcement learning approaches.

\section{CONCLUSION}

In this paper, we reported on a comparative user study researching the influence of proxemic-aware behavior (e.g., socially conform behavior body positioning and orientation) as an important factor for mobile companion robots being introduced to a new home. In our best of knowledge, our research addresses an important gap in research and demonstrates that proxemic behavior has the potential to improve in general the perception of mobile companion robots, considering four constructs measured by the Godspeed questionnaire (i.e., anthropomorphism, animacy, likability, and perceived intelligence). In addition, participants reported significant improvements considering perceived overall trustworthiness of the robot and willingness to interact again with the robot. All the results indicate that, as we expected and hypothesized proxemic-awareness improves the overall perceived (social and affective) quality of mobile companion robots.

\section{ACKNOWLEDGments}

The work described in this paper has been partially supported by the BMBF under 16SV7960 within the VIVA project. We thank Peter Fefelow, Lukas Huhn, and Isabella Kohlmus for their help conducting the user study.

\section{REFERENCES}

[1] C. Bartneck and J. Forlizzi, "A design-centred framework for social human-robot interaction," in RO-MAN 2004. 13th IEEE International Workshop on Robot and Human Interactive Communication. IEEE, 2004, pp. 591-594.

[2] T. Fong, I. Nourbakhsh, and K. Dautenhahn, "A survey of socially interactive robots," Robotics and autonomous systems, vol. 42, no. 34, pp. 143-166, 2003.

[3] M. W. Gamini Dissanayake, P. Newman, S. Clark, H. F. DurrantWhyte, and M. Csorba, "A solution to the simultaneous localization and map building (SLAM) problem," IEEE Transactions on Robotics and Automation, vol. 17, no. 3, pp. 229-241, 2001.

[4] E. T. Hall, The hidden dimension. Garden City, NY: Doubleday, 1910, vol. 609.

[5] M. L. Walters, D. S. Syrdal, K. L. Koay, K. Dautenhahn, and R. Te Boekhorst, "Human approach distances to a mechanical-looking robot with different robot voice styles," in Proceedings of the 17th IEEE International Symposium on Robot and Human Interactive Communication, RO-MAN, 2008, pp. 707-712.

[6] L. Takayama and C. Pantofaru, "Influences on proxemic behaviors in human-robot interaction," in 2009 IEEE/RSJ International Conference on Intelligent Robots and Systems, IROS 2009, 2009, pp. 5495-5502.

[7] G. Eresha, M. Haring, B. Endrass, E. Andre, and M. Obaid, "Investigating the influence of culture on proxemic behaviors for humanoid robots," in Proceedings - IEEE International Workshop on Robot and Human Interactive Communication, 2013, pp. 430-435.

[8] M. Obaid, E. B. Sandoval, J. Zlotowski, E. Moltchanova, C. A. Basedow, and C. Bartneck, "Stop! That is close enough. How body postures influence human-robot proximity," in 25th IEEE International Symposium on Robot and Human Interactive Communication, ROMAN 2016, 2016, pp. 354-361.

[9] J. Rios-Martinez, A. Spalanzani, and C. Laugier, "From Proxemics Theory to Socially-Aware Navigation: A Survey," International Journal of Social Robotics, vol. 7, no. 2, pp. 137-153, 2015.

[10] N. Marquardt and S. Greenberg, "Proxemic Interactions: From Theory to Practice," Synthesis Lectures on Human-Centered Informatics, 2015.

[11] F. Hemmert, M. Löwe, A. Wohlauf, and G. Joost, "Animate mobiles: Proxemically reactive posture actuation as a means of relational interaction with mobile phones," in Proceedings of the 7th International Conference on Tangible, Embedded and Embodied Interaction, ser. TEI '13. New York, NY, USA: ACM, 2013, pp. 267-270.

[12] E. W. Pedersen, S. Subramanian, and K. Hornbæk, "Is my phone alive?: A large-scale study of shape change in handheld devices using videos," in Proceedings of the 32Nd Annual ACM Conference on Human Factors in Computing Systems, ser. CHI '14. New York, NY, USA: ACM, 2014, pp. 2579-2588.

[13] I. Aslan and E. André, "Pre-touch proxemics: Moving the design space of touch targets from still graphics towards proxemic behaviors," in Proceedings of the 19th ACM International Conference on Multimodal Interaction, ser. ICMI '17. New York, NY, USA: ACM, 2017, pp. $101-109$.

[14] I. Aslan, B. Bittner, F. Müller, and E. André, "Exploring the user experience of proxemic hand and pen input above and aside a drawing screen," in Proceedings of the 17th International Conference on Mobile and Ubiquitous Multimedia. ACM, 2018, pp. 183-192.

[15] I. Aslan, T. Meneweger, V. Fuchsberger, and M. Tscheligi, "Sharing touch interfaces: Proximity-sensitive touch targets for tablet-mediated collaboration," in Proceedings of the 2015 ACM on International Conference on Multimodal Interaction. ACM, 2015, pp. 279-286.

[16] B. Bittner, I. Aslan, C. T. Dang, and E. André, "Of Smarthomes, IoT Plants, and Implicit Interaction Design," in Proceedings of the Thirteenth International Conference on Tangible, Embedded, and Embodied Interaction - TEI '19, 2019, pp. 145-154.

[17] C. Peters, S. Asteriadis, and K. Karpouzis, "Investigating shared attention with a virtual agent using a gaze-based interface," Journal on Multimodal User Interfaces, vol. 3, no. 1-2, pp. 119-130, 2010.

[18] N. Bee, E. André, and S. Tober, "Breaking the ice in human-agent communication: Eye-gaze based initiation of contact with an embodied conversational agent," in Lecture Notes in Computer Science (including subseries Lecture Notes in Artificial Intelligence and Lecture Notes in Bioinformatics), vol. 5773 LNAI, 2009, pp. 229-242.

[19] J. Li, A. Cuadra, B. Mok, B. Reeves, J. Kaye, and W. Ju, "Communicating dominance in a nonanthropomorphic robot using locomotion," ACM Trans. Hum.-Robot Interact., vol. 8, no. 1, pp. 4:1-4:14, Mar. 2019. 
[20] T. M. Ciolek and A. Kendon, "Environment and the Spatial Arrangement of Conversational Encounters," Sociological Inquiry, vol. 50, no. 3-4, pp. 237-271, 1980.

[21] I. Gaudiello, E. Zibetti, S. Lefort, M. Chetouani, and S. Ivaldi, "Trust as indicator of robot functional and social acceptance. An experimental study on user conformation to iCub answers," Computers in Human Behavior, vol. 61, pp. 633-655, 2016.

[22] M. Salem, G. Lakatos, F. Amirabdollahian, and K. Dautenhahn, "Would You Trust a (Faulty) Robot?" pp. 141-148, 2015.

[23] R. Beckers, O. Holland, and J.-L. Deneubourg, "From local actions to global tasks: Stigmergy and collective robotics," in Artificial life IV, vol. 181, 1994, p. 189.

[24] J.-L. Deneubourg, S. Goss, N. Franks, A. Sendova-Franks, C. Detrain, and L. Chrétien, "The dynamics of collective sorting robot-like ants and ant-like robots," in Proceedings of the first international conference on simulation of adaptive behavior on From animals to animats, 1991, pp. 356-363.

[25] K. Dautenhahn and A. Billard, "Bringing up robots or-the psychology of socially intelligent robots: From theory to implementation," in International Conference on Autonomous Agents: Proceedings of the third annual conference on Autonomous Agents, vol. 1999. Citeseer, 1999, pp. 366-367.

[26] M. Coeckelbergh, "Can we trust robots?" Ethics and information technology, vol. 14, no. 1, pp. 53-60, 2012.

[27] G. M. Maxwell, M. W. Cook, and R. Burr, "The encoding and decoding of liking from behavioral cues in both auditory and visual channels," Journal of Nonverbal Behavior, vol. 9, no. 4, pp. 239-263, 1985.

[28] K. R. MacArthur, K. Stowers, and P. Hancock, "Human-robot interaction: Proximity and speed-slowly back away from the robot!" in Advances in Human Factors in Robots and Unmanned Systems. Springer, 2017, pp. 365-374.

[29] M. L. Walters, M. A. Oskoei, D. S. Syrdal, and K. Dautenhahn, "A long-term human-robot proxemic study," in 2011 RO-MAN. IEEE, 2011, pp. 137-142.

[30] S. S. Honig, T. Oron-Gilad, H. Zaichyk, V. Sarne-Fleischmann, S. Olatunji, and Y. Edan, "Toward socially aware person-following robots," IEEE Transactions on Cognitive and Developmental Systems, vol. 10, no. 4, pp. 936-954, 2018.
[31] J. D. Lewis and A. Weigert, "Trust as a social reality," Social forces, vol. 63 , no. 4, pp. 967-985, 1985.

[32] C. Castelfranchi and R. Falcone, Trust theory: A socio-cognitive and computational model. John Wiley \& Sons, 2010, vol. 18.

[33] C. Bartneck, E. Croft, and D. Kulic, "Measurement instruments for the anthropomorphism, animacy, likeability, perceived intelligence, and perceived safety of robots," International Journal of Social Robotics, vol. 1, no. 1, pp. 71-81, 2009.

[34] S. Holm, "A simple sequentially rejective multiple test procedure," Scandinavian journal of statistics, pp. 65-70, 1979.

[35] L. V. Hedges, "Distribution theory for glass's estimator of effect size and related estimators," journal of Educational Statistics, vol. 6, no. 2, pp. 107-128, 1981

[36] L. Hedges and I. Olkin, Statistical Methods for Meta-Analysis. London: Academic Press., 1985.

[37] J. Cohen, Statistical power analysis for the behavioral sciences, 2nd ed. Hillsdale, NJ [u.a.]: Erlbaum, 1988.

[38] B. R. Duffy, "Anthropomorphism and the social robot," Robotics and autonomous systems, vol. 42, no. 3-4, pp. 177-190, 2003.

[39] B. Kuipers, "How can we trust a robot?" Communications of the ACM, vol. 61, no. 3, pp. 86-95, 2018.

[40] H. Ritschel, T. Baur, and E. André, "Adapting a robot's linguistic style based on socially-aware reinforcement learning," in 26th IEEE International Symposium on Robot and Human Interactive Communication, RO-MAN 2017, Lisbon, Portugal, August 28 - Sept. 1, 2017, 2017, pp. 378-384. [Online]. Available: https://doi.org/10.1109/ROMAN.2017.8172330

[41] H. Ritschel, A. Seiderer, K. Janowski, I. Aslan, and E. André, "Drink-o-mender: An adaptive robotic drink adviser," in Proceedings of the 3rd International Workshop on Multisensory Approaches to Human-Food Interaction, MHFI@ICMI 2018, Boulder, CO, USA, October 16, 2018, 2018, pp. 3:1-3:8. [Online]. Available: https://doi.org/10.1145/3279954.3279957

[42] K. Weber, H. Ritschel, I. Aslan, F. Lingenfelser, and E. André, "How to shape the humor of a robot - social behavior adaptation based on reinforcement learning," in Proceedings of the 20th ACM International Conference on Multimodal Interaction, ser. ICMI '18. New York, NY, USA: ACM, 2018, pp. 154-162. 